Commun. Fac. Sci. Univ. Ank. Ser. A1 Math. Stat.

Volume 69, Number 1, Pages $717-738(2020)$

DOI: $10.31801 /$ cfsuasmas. 567078

ISSN 1303-5991 E-ISSN 2618-6470

http://communications.science.ankara.edu.tr/index.php?series=A1

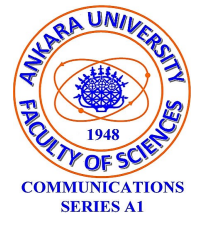

\title{
AN EVALUATION OF SOME METHODS USED FOR DETERMINATION OF HOMOGENOUS STRUCTURAL BREAK POINT IN MEAN OF PANEL DATA
}

\author{
SELIM DAĞLIOĞLU AND M. AKIF BAKIR
}

\begin{abstract}
In this study, performances of correct break point estimation of Simple Mean Shift Model Method, Fluctuation Test, Wald Statistic Test and Kim Test methods used to investigate presence of structural break and determine the date of break in a panel data consisting of $\mathrm{N}$ time series, each of $\mathrm{T}$ length, belonging to $\mathrm{N}$ cross-section have been investigated. In this context, 108 Monte Carlo simulations with each 3000 repeats have been carried out for $3,3,4$ and 3 levels of factors, respectively number of cross-section units, length of series, size of break and proportion of break, to evaluate the performance of these tests used for determination of structural break in panel data. According to the Monte Carlo simulations it is concluded that Simple Mean Shift Model approach has better performance of break point estimation than other methods. Moreover, while Wald Test puts forth its best performance in the case where the breaks in series are at the half of the series, Fluctuation and Kim Tests showed their best performances in the case that the breaks are at the third quarter of series. Generally, correct break point estimation performances of tests decrease as the number of cross-section or length of series increases, even if it is limited. The changes at the levels of the proportion of break factor also lead to high accuracy estimation performance of different methods. Moreover, increases at the size of break usually decreases rates of correct estimation of methods and they approach to zero while means of the series changed $40 \%$ and over after break.
\end{abstract}

\section{INTRODUCTION}

Structural break(s) is(are) permanent change(s) in the structure of variables, due to permanent effects of economic or financial shocks, policy changes, cultural and technological changes, etc., on the distribution of variables. Changes in the behaviour of economic time series such as employment, growth and unemployment can occur in the long run due to policy changes and various economic events.

Received by the editors: May 13, 2019; Accepted: January 23, 2020.

2010 Mathematics Subject Classification. Primary 05C38, 15A15; Secondary 05A15, 15A18.

Key words and phrases. Panel data, structural break point, correct estimation of break point, Monte Carlo simulation.

(C)2020 Ankara University Communications Faculty of Sciences University of Ankara-Series A1 Mathematics and Statistics 
However, when the models used in examining the data for such variables are estimated, it is usually assumed that the model parameters do not change over the sampling periods. This assumption makes the analysis relatively simple. However, the assumption that a time series is not subject to a change throughout the sample becomes more difficult to achieve as the length of the series increases. In the case of structural breaks in series, continuing analysis without considering this structural change can lead to incorrect estimations of model parameters. A typical example of this is that the investigation of the presence of unit root in Nelson and Plosser data; Nelson and Plosser [1], Perron [2], Zivot and Andrews [3] and Lumsdaine and Papell [4] have achieved different results. Despite the use of the same data set in these studies, the results differ depending on whether structural breaks are taken into account and whether structural breaks are included in the model.

The time series consists of observations obtained over a single cross-sectional unit at different times. Policy or technology changes often lead to permanent changes in the structure of the time series. For this reason, structural breaks are often encountered in time series. However, some difficulties arise when estimating the break point in the time series. If a structural break occurs at any time point $k_{0}$ of time series $y_{t}$, the break point $k_{0}$ can not be consistently predicted, regardless of how large the sample is, and the estimator $\hat{k}$ of the break point $k_{0}$ is not consistent. Therefore, it is usually attempted to estimate the break fraction instead of estimating the $k_{0}$ 's in which the structural change occurs in the time series. The effectiveness of the approach using a single time series in determining structural break depends on two assumptions: First, the magnitude of structural break (the difference between pre-break mean and post-break mean) is large enough. The second is that the true point of break point $k_{0}$ is far enough from the beginning and end of the sample. In a single series it is impossible to identify break point when the regime has a single observation [5], [6]. In the study of both single and multiple structural break points in time series, asymptotic framework is used in which the magnitudes of change(s) asymptotically converge to zero as the sample size increases in order to obtain critical statistics [7]. In other words, obtaining the limit distribution of the test statistics requires the assumption that the size of the structural break decreases as the sample size increases [8]. In the structural break literature this assumption is called the shrinking magnitude of structural break assumption. According to this assumption, as the sample size increases in the time series, the break point can be determined [9]. Both the break point inconsistency and the necessity of reduced break are related to the problem of defining the break point in time series models. The main reason for these two situations to emerge is that time series can not carry enough information. Additional information is needed in order to determine the actual break point in the time series. This information is tried to be obtained by increasing the sample size. When examining structural break in panel data, the additional information carried by the cross-sectional dimension of panel data eliminate the necessity of artificially increasing the number of observations using the 
reduced shrinking magnitude of structural break assumption. In addition, panel data can be used to derive asymptotics around the actual break date, since it has the cross-sectional dimension as well as the time dimension [9].

Although methods using panel data have significant advantages when compared to methods using only time series or only cross-sectional data, methods using panel data are much more complex. In this context, different methods for determining structural break point in panel data have been developed in the structural break literature. It has become widespread that structural break problem has been examined in panel data in recent years. The studies on the structural break problem in the panel data are generally considered in two directions. The first is to investigate the existence of unit root in panel data in the presence of structural break. The second is to determine the existence and date of structural break point. Two approaches have been adopted in panel studies in relation to the assumptions made about the position of structural break data. While the first considers the assumption that structural breaks in all series of the panel have emerged in a common date, in the second approach break point is assumed to be random in which break point occur on a different date for each series depending on the distribution of the random variable. The methods assuming the random break point are more complicated than the methods considering the common break point hypothesis.

The assumption of the common break point has been used in the studies by Han and Park [10], Joseph and Wolfson [11], Bai [12], Bai et al. [13], Emerson and Kao [14], Bai and Perron [15], Kao et. al. [16], Feng et. al. [9], Kim [17], Horváth and Hušková [18], Chan et. al. [19] and Li et. al. [20]. On the other hand, the assumption of random break point is considered in studies such as Joseph and Wolfson [11], Joseph and Wolfson [21], Joseph, Vandal and Wolfson [22], Joseph at al. [23], Joseph at al. [24] and Liao [6].

While there have been various methods developed in the literature on structural breaks in panel data, no study has been found on the comparison of the performance of these methods in the context of determining break point [25]. The contribution of this study is to compare the correct break point estimation performance of some methods used to determine the structural break point under the assumption of the common break point, according to the factors the number of cross sections, time series dimension, break size and break fraction. In this context, with the aid of Monte Carlo simulations, the Simple Mean Shift Model Method proposed in Bai [5], the Fluctuation Test and the Wald Statistic Test proposed in Emerson and Kao [14] and the Kim Test proposed in Kim [17] performance are evaluated.

In the next section of the study, the performances of the considered methods estimating the breakpoint are discussed. In the third section of the study, the data generating process and the issues considered in determination of factor levels and the assumption of Monte Carlo simulation are explained. In the fourth part of the study, the results obtained by Monte Carlo simulations are given. In the fifth and 
last part, the results obtained in the study are discussed and some suggestions are made.

\section{Methods for Determination of Break point}

Bai [5] considers the following simple mean shift model:

$$
\begin{array}{ll}
y_{i t}=\mu_{i 1}+u_{i t} & t=1,2, \ldots, k_{0} \\
y_{i t}=\mu_{i 2}+u_{i t} & t=k_{0}+1, \ldots, T .
\end{array}
$$

where $E\left(u_{i t}\right)=0$ for all $i$ and $t$. In this model, each series has a break point at $k_{0}$, where $k_{0}$ is unknown. The $\mu_{i 1}$ and $\mu_{i 2}$ are pre-break mean and post-break mean of $y_{i t}$, respectively. For the simple mean shift model, he proposes the OLS estimator of $k_{0}$ as in Equation 2.

$$
\hat{k}=\underset{1 \leq k \leq T-1}{\arg \min } S S R(k) .
$$

where sum of square of residuals $S S R_{i T}(k)$ is

$$
\operatorname{SSR}_{i T}(k)=\left\{\begin{array}{cl}
\sum_{t=1}^{k}\left(y_{i t}-\bar{y}_{i 1}\right)^{2}+\sum_{t=k+1}^{T}\left(y_{i t}-\bar{y}_{i 2}\right)^{2} & , k=1,2, \ldots, T-1 \\
\sum_{t=1}^{T}\left(y_{i t}-\bar{y}_{i}\right)^{2} & , \quad k=T
\end{array}\right.
$$

for each $k=1,2, \ldots, T$. Also $\bar{y}_{i}$ is the average of all the observations of cross-section unit defined by,

$$
\begin{aligned}
& \bar{y}_{i 1}=\frac{1}{k} \sum_{t=1}^{k} y_{i t} \\
& \bar{y}_{i 2}=\frac{1}{T-k} \sum_{t=k+1}^{T} y_{i t}
\end{aligned}
$$

and sum of residual squares over all equations is as in Equation 5

$$
S S R(k)=\sum_{i=1}^{N} S S R_{i T}(k) .
$$

Emerson and Kao [14] consider the one-way random effect panel regression model with the deterministic time trend given in Equation 6

$$
\begin{aligned}
& y_{i t}=\alpha+\beta_{t} X_{t}+v_{i t} \\
& v_{i t}=\mu_{i}+u_{i t} .
\end{aligned}
$$

where $\beta$ is the slope parameter, $X_{t}=\frac{t}{T}$, unobservable individual effects are $\mu_{i} \sim$ $i i d\left(0, \sigma_{\mu}^{2}\right)$ and disturbance term of $\operatorname{AR}(1)$ is $u_{i t}=\rho u_{i t-1}+\varepsilon_{i t}, \varepsilon \sim i i d\left(0, \sigma_{\varepsilon}^{2}\right)$. They propose two different methods for testing the following null hypothesis

$$
H_{0}: \beta_{t}=\beta ; \forall t \in[1, T]
$$


meaning that there is no change in the model against the following alternative hypothesis

$$
H_{1}: \beta_{t}=\left\{\begin{array}{lll}
\beta_{1} & , \quad t=1,2, \ldots, k \\
\beta_{2} & , \quad t=k+1, \ldots, T
\end{array}\right.
$$

meaning that there exists a change in the $k$ - point. They proposed to estimate the break point according to these two methods, The first is based on the fluctuation test of Ploberger, Kramer and Kontrus [26], while the second one is based on the mean statistics of Andrew and Ploberger [27] and exponential Wald statistic and the Wald statistic of Andrew [28]. In testing null hypothesis with fluctuation test, if the difference

$$
\max _{i=1, \ldots, k}\left|\hat{\beta}_{k}-\hat{\beta}_{T}\right|
$$

is big enough, that is when $\hat{\beta}_{k}$ is too much fluctuating, the null hypothesis is rejected. In other words, there is a structural break at this point and $\left|\hat{\beta}_{k}-\hat{\beta}_{T}\right|$ is the estimate of the break point. In Equation $(9), \hat{\beta}_{T}$ denotes the estimate of the slope parameter over all panel data estimated by OLS method, and $\hat{\beta}_{k}$, which is estimated with recursive OLS, is

$$
\hat{\beta}_{k}=\frac{\sum_{i=1}^{N}\left[\sum_{t=1}^{k}\left(X_{t}-\bar{X}_{k}\right) y_{i t}\right]}{\sum_{i=1}^{N} \sum_{t=1}^{k}\left(X_{t}-\bar{X}_{k}\right)^{2}}
$$

where

$$
\bar{X}_{k}=\frac{1}{k} \sum_{t=1}^{k} X_{t} .
$$

In the Wald statistic test, the break point is estimated to be

$$
\hat{k}=\underset{\left[T r^{+} \leq k \leq T-\left[T r^{+}\right]\right]}{\arg \min } W_{1}(k) .
$$

Here,

$$
\tilde{\sigma}_{u}^{2}=\frac{1}{N T} \sum_{i=1}^{N} \sum_{t=1}^{T}\left(v_{i t}-\bar{v}_{i}\right)^{2}
$$

and the estimation of $\sigma_{0}^{2}$ is

$$
\sigma_{0}^{2}=\frac{\sigma_{\varepsilon}^{2}}{(1-\rho)^{2}}
$$

Thus,

$$
W_{1}(k)=\frac{\tilde{\sigma}_{u}^{2}}{3 \sigma_{0}^{2}} W(k) .
$$


In addition,

$$
\begin{gathered}
\hat{\beta}_{1 k}=\frac{\sum_{i=1}^{N}\left[\sum_{t=1}^{k}\left(X_{t}-\bar{X}_{1 k}\right) y_{i t}\right]}{\sum_{i=1}^{N} \sum_{t=1}^{k}\left(X_{t}-\bar{X}_{1 k}\right)^{2}} \\
\hat{\beta}_{2 k}=\frac{\sum_{i=1}^{N}\left[\sum_{t=k+1}^{T}\left(X_{t}-\bar{X}_{2 k}\right) y_{i t}\right]}{\sum_{i=1}^{N} \sum_{t=k+1}^{T}\left(X_{t}-\bar{X}_{2 k}\right)^{2}} \\
\bar{X}_{1 k}=\frac{1}{k} \sum_{t=1}^{k} X_{t}
\end{gathered}
$$

and

$$
\bar{X}_{2 k}=\frac{1}{T-k} \sum_{t=k+1}^{T} X_{t} .
$$

Then $W(k)$ is calculated as follows:

$$
W(k)=\frac{1}{\hat{\sigma}_{u}^{2}} \frac{\left(\hat{\beta}_{1 k}-\hat{\beta}_{2 k}\right)^{2}}{\left[\left(\sum_{i=1}^{N} \sum_{t=1}^{k}\left(X_{t}-\bar{X}_{1 k}\right)^{2}\right)^{-1}+\left(\sum_{i=1}^{N} \sum_{t=k+1}^{T}\left(X_{t}-\bar{X}_{2 k}\right)^{2}\right)^{-1}\right]} .
$$

Kim [17] considers the model with the deterministic trend and the disturbance component given in Equation 18 .

$$
y_{i t}=d_{i t}+u_{i t}, \quad i=1, \ldots, N \text { and } t=1, \ldots, T .
$$

The deterministic component $d_{i t}$ can be considered in three different ways to be

$$
d_{i t}=\left\{\begin{array}{cll}
\mu_{i}+\beta_{i} t+\gamma_{i} B_{t} & , & \text { Model I (Joint broken trend) } \\
\mu_{i}+\beta_{i} t+\theta_{i} C_{t}+\gamma_{i} B_{t} & , & \text { Model II (Locally broken trend) } \\
\mu_{i}+\beta_{i} t+\theta_{i} C_{t} & , & \text { Model III (Mean shift) }
\end{array}\right.
$$

where

and

$$
C_{t}=\left\{\begin{array}{lll}
0, & t \leq k_{0} \\
1, & t>k_{0}
\end{array}\right.
$$

$$
B_{t}=\left(t-k_{0}\right) C_{t} .
$$

Here, Equation 20 can be rewritten for all of three models, if $t \leq k_{0}$, then $d_{i t}=$ $\mu_{i}+\beta_{i} t$ and if $t>k_{0}$, then

$$
d_{i t}=\left\{\begin{array}{cll}
\mu_{i}-k_{0} \gamma_{i}+\left(\beta_{i}+\gamma_{i}\right) t & , & \text { Model I (Joint broken trend) } \\
\mu_{i}-k_{0} \gamma_{i}+\theta_{i}+\left(\beta_{i}+\gamma_{i}\right) t & , & \text { Model II (Locally broken trend) } \\
\mu_{i}+\beta_{i} t+\theta_{i} & , & \text { Model III (Mean shift) }
\end{array}\right.
$$


Models I and II are extended form of the panel data models reviewed by Perron and Zhu [29] for the univariate case. Model III, on the other hand, is an extended form so as to include a deterministic trend of the mean shift model examined in Bai [5].

The regression coefficients in the model are not restricted to be common for each section. For this reason, instead of estimating the regression coefficients jointly by pooling the cross-section data, the regression coefficients can be estimated separately for each equation using the OLS method. Thus, in the Kim Test, the individual OLS estimators of the regression coefficients for each equations are used for each cross section unit [17].

The Kim test assumes that the actual break point is unknown and the break fraction defined to be $\lambda_{1}=k_{0} / T ; \quad \lambda_{1} \in[\pi, 1-\pi], \quad \pi \in(0,1 / 2)$ is constant for every $T$. It is also assumed that the break point $k_{0}$ is common to all equations and that the break fraction $\lambda_{1}$ remains constant as the sample size grows.

Using the deterministic time trend definitions given in Equation (19), the model in Equation (18) can be rewritten with matrix notation for each equation as

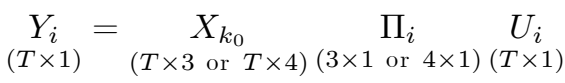

where $Y_{i}=\left(y_{i 1}, \ldots, y_{i T}\right)^{\prime}$ and $U_{i}=\left(u_{i 1}, \ldots, u_{i T}\right)^{\prime}$. The variables and coefficients of Equation 22 are defined as follows:

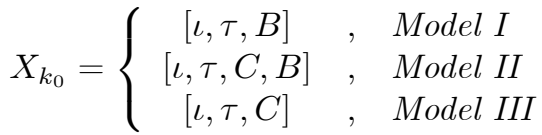

$$
\begin{aligned}
& \Pi_{i}=\left\{\begin{array}{cll}
\left(\mu_{i}, \beta_{i}, \gamma_{i}\right)^{\prime} & , & \text { Model I } \\
\left(\mu_{i}, \beta_{i}, \theta_{i}, \gamma_{i}\right)^{\prime} & , & \text { Model II } \\
\left(\mu_{i}, \beta_{i}, \theta_{i}\right)^{\prime} & , & \text { Model III }
\end{array}\right.
\end{aligned}
$$

where $\iota=(1, \ldots, 1)^{\prime}, \tau=(1, \ldots, T)^{\prime}, C=\left(C_{1}, \ldots, C_{T}\right)^{\prime}, B=\left(B_{1}, \ldots, B_{T}\right)^{\prime}, X_{k_{0}}$ is the collection of all dependent variables, and $\Pi_{i}$ is the regression coefficient for the corresponding equation.

Then, the whole $N$ equation system can be written as

$$
Y=X_{k_{0}} \Pi+U
$$

where $Y=\left[Y_{1}, \ldots, Y_{N}\right], \Pi=\left[\Pi_{1}, \ldots, \Pi_{N}\right]$ and $U=\left[U_{1}, \ldots, U_{N}\right]$. Also the row vectors are defined as $\mu=\left(\mu_{1}, \ldots, \mu_{N}\right), \beta=\left(\beta_{1}, \ldots, \beta_{N}\right), \theta=\left(\theta_{1}, \ldots, \theta_{N}\right)$ and $\gamma=$ $\left(\gamma_{1}, \ldots, \gamma_{N}\right)$. Then, an alternative expression for $\Pi$ is $\left[\mu^{\prime}, \beta^{\prime}, \gamma^{\prime}\right]^{\prime},\left[\mu^{\prime}, \beta^{\prime}, \theta^{\prime}, \gamma^{\prime}\right]^{\prime}$ and $\left[\mu^{\prime}, \beta^{\prime}, \theta^{\prime}\right]^{\prime}$ for Model I, II and III, respectively.

A general break point and a general break fraction are denoted by $k$, and $\alpha=$ $k / T$, respectively, and $X_{k}$ is defined similarly to $X_{k_{0}}$. Then, the sum of residual squares for each $k$, can be defined as follows:

$$
S S R(k)=\operatorname{tr}\left[Y^{\prime}\left(I-P_{k}\right) Y\right]
$$


where $P_{k}=X_{k}\left(X_{k}^{\prime} X_{k}\right)^{-1} X_{k}^{\prime}$ and $\operatorname{tr}[$.$] is trace operator. Thus, estimated break$ point is the one minimizing the sum of residual squares such as

$$
\hat{k}=\underset{k}{\arg \min } S S R(k)
$$

and

$$
\hat{\lambda}=\frac{\hat{k}}{T}
$$

\section{Data generation and Monte-Carlo simulations}

In this section, we evaluate the correct estimation performance of the Simple Mean Shift Model Method (hereafter referred to as Bai Test) proposed by Bai [5], the Fluctuation Test, Wald Statistic Test (hereafter referred to as Wald test) and Kim Test proposed in Kim [17], for the break date with Monte Carlo simulations.

The panel data to which the tests are applied are generated in accordance with the following model, also given in Equation (1):

$$
\begin{aligned}
& y_{i t}=\mu_{i 1}+u_{i t}, t=1,2, \ldots, k_{0} \\
& y_{i t}=\mu_{i 2}+u_{i t}, t=k_{0}+1, \ldots, T
\end{aligned}
$$

where, $i=1,2, \ldots, N, t=1,2, \ldots, T, y_{i t}$ is the observation value of the $i$ th section unit at time $t, \mu_{i 1}$ is the pre-break mean which is mean of the panel data before the break date, $\mu_{i 2}$ is the post-break mean which is mean of the panel data after the break date, $k_{0}$ is the common break point and $u_{i t}$ indicates the disturbance terms. In the simulations, the disturbance terms are generated from independent and identically distributed $u_{i t} \stackrel{i i d}{\sim} N(0 ; 1)$, and, $\mu_{i 1}$ and $\mu_{i 2}$ are from $\mu_{i 1} \stackrel{i i d}{\sim} N(3 ; 0,24)$ and $\mu_{i 2} \stackrel{i i d}{\sim} N(3 \times \gamma ; 0,24)$ where $\gamma$ denotes the break ratio.

The number of repetitions are decided by taking into account the difference between asymptotics of the estimated break points in sequential run of simulations. In the study, the number of repetitions was determined as 3000 runs with the difference 0,001 between the average values of the break points predicted in each repetition. In total, Monte Carlo simulations are repeated as many times as the number of factor combinations depending on the level of the four factors under investigation.

Various issues have been taken into account to determine the factor levels. These issues can be summarized as follows: When examining the effects of time and crosssection length on break point estimation performance, the levels of these factors are defined as small, medium and large. The levels are chosen as 12, 32 and 120 for both time dimension $T$ and cross-sectional dimension $N$.

If the break point $k_{0}$ is defined as a set of fixed values, the marginal effect of the break point can not be observed due to the coexistence of changes in the break point at different time dimension and the effects of changes in time dimension. For this reason, instead of taking the break point $k_{0}$ as a member of a fixed value set in simulations, $k_{0}$ is defined as an integer between 1 and $T, k_{0}=[T \lambda], \lambda \in(0,1)$. 
Thus, in the simulations, breaks are allowed to occur in the first, second and third quarter of the panel data, respectively, taking into account $\lambda \in\{0,25 ; 0,50 ; 0,75\}$ to define the break fraction.

The final factor by which the effect on the break point estimation performance is investigated is the magnitude of the break $\left(\mu_{i 2}-\mu_{i 1}\right)$. When the magnitude of break factor levels are determined, the post-break mean is defined as

$$
\mu_{i 2}=\mu_{i 1} \times \gamma
$$

where $\gamma$ is the break ratio. Then, the magnitude of the break is constant and written in the following form:

$$
\left(\mu_{i 2}-\mu_{i 1}\right)=\gamma \times \mu_{i 1}-\mu_{i 1}=(\gamma-1) \mu_{i 1}
$$

Thus, the magnitude of the break is defined as the ratio of the pre-break mean. Expression of the magnitude of break in this way allows it to be fixed for different factor levels and to define the post-break mean to be smaller than the pre-break mean. For this reason, when examining the effect of magnitude of break on the performance of the tests, the break ratio factor, $\gamma$, is strictly defined as the pre-break mean is used. The levels of the break ratio are defined as $\gamma \in\{1,1 ; 0,8 ; 1,4 ; 1,9\}$ so as to include the case where the post-break panel mean is smaller than the pre-break panel mean.

Simulation is performed at a total of 108 points of the experimental design for the factors time dimension, cross-section dimension, break fraction and break ratio, with the levels $3,3,3$ and 4 , respectively.

\section{Simulation Results}

In this section, the simulation results obtained via the simulation design described in the third section about correct break point estimation performance of the Bai, Fluctuation, Wald, and Kim tests are given. After generation of panel data, indicator variable is generated by using break point estimates of Bai, Fluctuation, Wald and Kim Tests to estimate correct estimation rates. Indicator variable shows whether estimated break point is equal actual break point or not. Indicator variable defined as dummy variable is given below:

$$
D_{j}=\left\{\begin{array}{lll}
1 & , & \hat{k}_{j}=k_{0} \\
0 & , & \hat{k}_{j} \neq k_{0}
\end{array}\right.
$$

where $j(j=1, \ldots, 4)$ shows the method used for estimating break point. This variable can take two values as 0 or 1 . Since the mean of the indicator variable is on the $[0,1]$ interval, it shows the correct estimation rate of tests under the certain factor assumptions. These rates have been used for evaluating the performance for correct estimation of tests. Figure 1 shows the the effects of the changes in the cross-section dimension on the correct estimation rates of the tests for different time dimension in the case that the breakpoint occurs at the first quarter of the series of the panel data and mean of the series decreases by $\% 20$ after break. When 

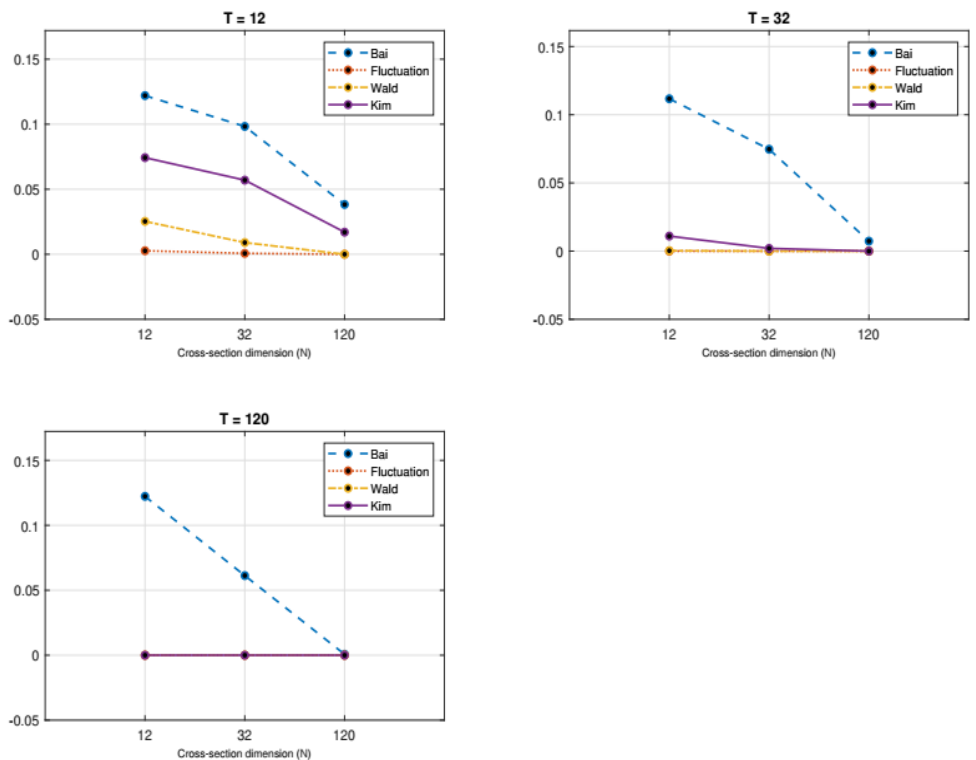

Figure 1. Simulation results for correct break point estimation rates $(\gamma=0,8$ and $\lambda=0,25)$

the break fraction and the break ratio factor are fixed, correct estimation rates of test convergences to zero by decreasing as both time and cross-section dimensions increase. Nevertheless, it is seen that decreasing at the rate of correct break point estimation are small. Moreover, while in the case where $T=12$, correct estimation rates of the Bai and Kim tests are different from zero, they converge to zero as the time and cross-section dimensions increase. Correct estimation rates of the tests, except the Bai's, are zero for the bigger factor levels of $T$.If a break occurs in the first quarter of the series and the mean of the series increases by $40 \%$ after break, the correct break point estimation rates of all the tests decrease as the cross-sectional dimension increases under different time dimensions. Compared to the case where the mean of the series is reduced by $20 \%$, the correct estimation rates of the tests show a similar tendency. Nevertheless, in the current case, the correct break points estimation rates of the tests are generally lower for all levels of the cross-sectional dimension. In other words, an increase in the rate of break ratio causes to a decrease in the correct estimation rates of the methods. In both cases, the highest correct estimation rates are achieved with the Bai Test. In the case where a break in the first quarter of the series and an $10 \%$ increase in the mean of the series after break, the correct break point estimation rates of all the tests, except rates of Bai Test, decreases as the cross-section size increases for different time dimensions. In panel data, the Bai Test correct estimation rates increase (Figure 3). However, when 

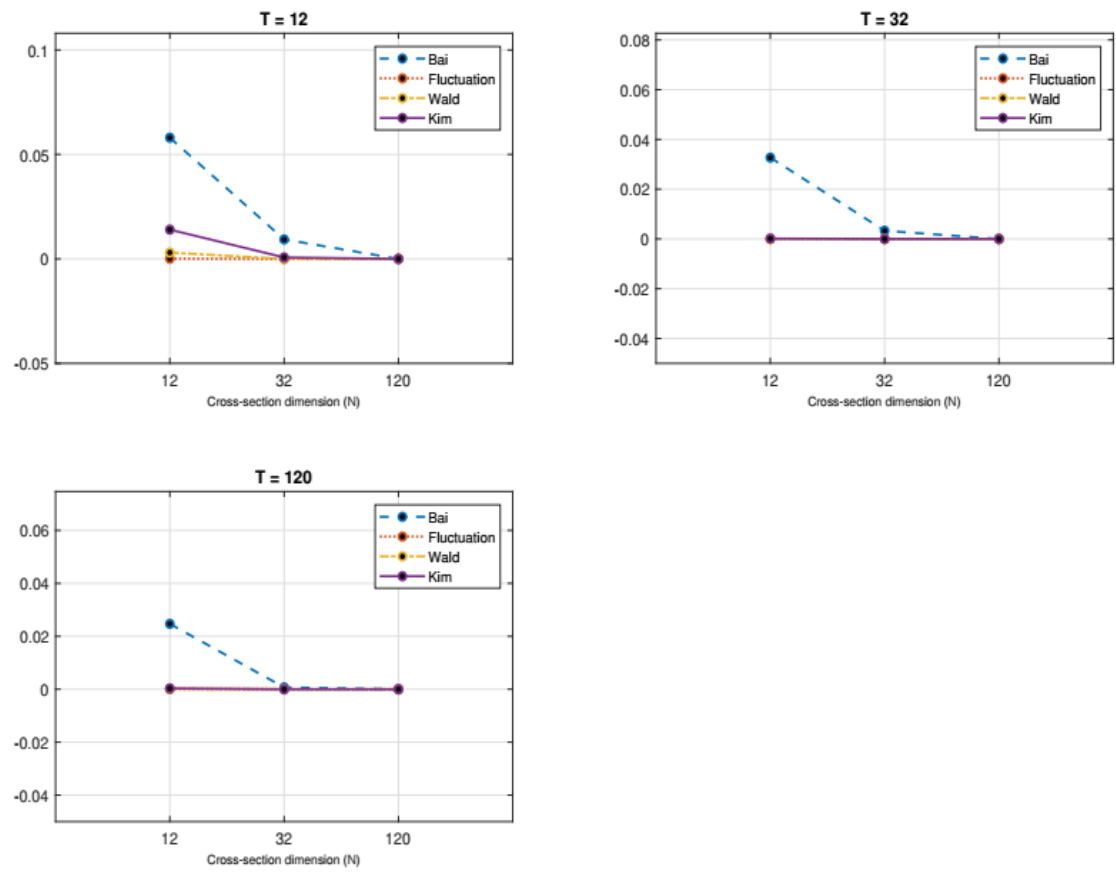

FiguRE 2. Simulation results for correct break point estimation rates $(\gamma=1,4$ and $\lambda=0,25)$

Figures 1 and 3 are evaluated jointly, the correct break point estimation rates of the test converges each other for both break ratios of $10 \%$ and $20 \%$.

Figures 1, 2 and 3 show that increments in the cross-section dimensions have an effects towards decreasing the correct estimation rates of break points. A similar situation is observed for time dimension and the break ratio. In other words, according to the results obtained in Figures 1,2 and 3, it can be said that the increases in the cross section and time dimension and the increase in the break ratio in the series have negative effect on the correct break points estimation rates of the tests. Figure 4 shows the effects of the break fraction on the correct break point estimation rates of Bai, Fructuation, Wald and Kim Tests when the time dimension and cross section size are fixed at 12. From the Figure 4, it is seen that the Wald Test has the highest correct estimation rate in the case that the breaks occur at the midpoint of the series. In addition, if the breaks occur in the later periods of the series, the correct break point estimation rates of the Fluctuation test increase. The Fluctuation Test shows highest correct estimation rates in the panels where break occurs in the second half of the series and the break ratio is small. Compared to other methods, the Bai Test has a generally high correct estimation 

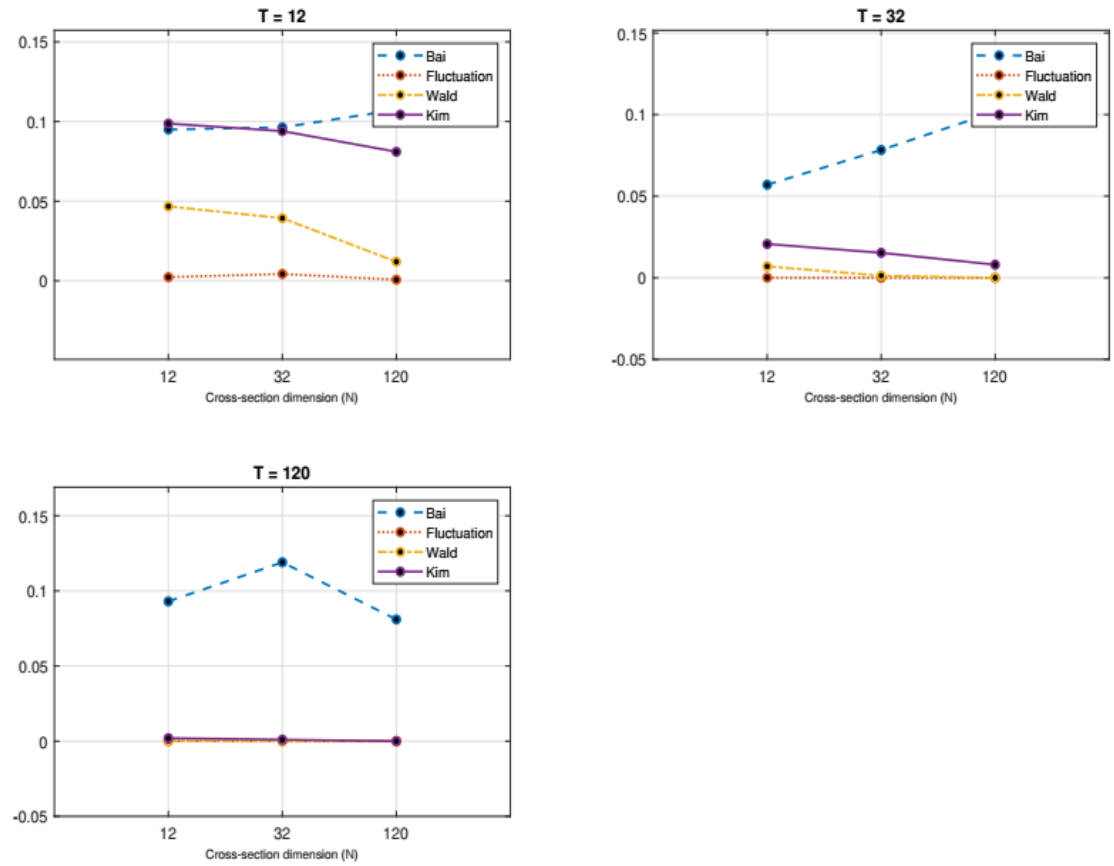

FiguRE 3. Simulation results for correct break point estimation rates $(\gamma=1,1$ and $\lambda=0,25)$

rate and they are less influenced by break fraction. In addition, Kim Test provides higher correct break point estimation rates if the break occurs in the later stages of the series similar to Fluctuation test.

An important finding emerging from Figure 4 is that the changes in the break fraction have effects on the correct estimation rates of the break point of the tests at different ways. Moreover, changes in break fraction has limited effects on correct break point estimation rates of the tests. Figure 5 shows the effects of the changes in the break ratio on the correct break point estimation rates of tests when the break occurred in first quarter, half or the third quarter of panel data formed by $N=32$ and $T=12$. In general, the correct break point estimation rate of the methods decreases as the break ratio increases.

In addition, if the post-break mean is $40 \%$ or more bigger than the pre-break mean, methods with some exceptions can not accurately estimate the break point depending on the break fraction in general. According to the region where the breaks occur in the series, the test having the highest correct break point estimation rate varies. In the case where the break occurs in the first, second and third quarter of the series, the Bai, Wald and Kim tests have the highest correct estimation rates, 

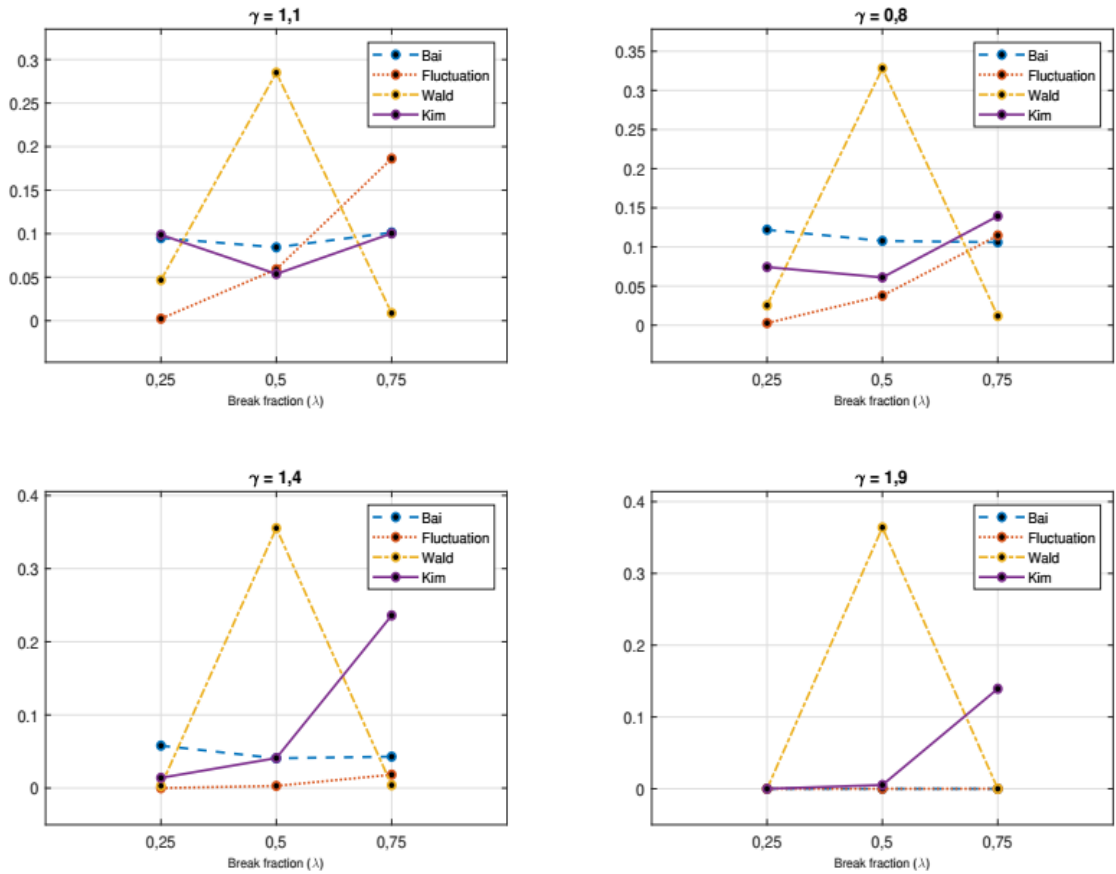

FiguRE 4. Simulation results for correct break point estimation rates $(N=12$ and $T=12)$

in order. Nevertheless, in the case where the break occurs in the third quarter of the series and the break ratio is small, the Fluctuation Test has the highest correct break point estimation rate. While the changes in the break fraction have a limited effect on the correct break point estimation rates of the tests except Wald test, they lead to change the tests having the highest correct break point estimation rates based on the occurrence of break in different regions of the series. Figure 6 shows the effects of changes in panel time dimension on the correct break point estimation rates of Bai, Fluctuation, Wald and Kim Tests where the mean of the series is reduced by $20 \%$ compared to the pre-break mean in the panel data consisting of 32 cross-sectional units. From Figure 6 it is seen that Bai Test mostly has higher correct estimation rates than others. Furthermore, the change in the break fraction leads to significant changes in the correct estimation rates of the tests. When the break occurs in the middle of the series, the Wald Test has higher correct estimation rates than others for $T=12$, whereas in the third quarter of the series, the Kim Test has higher correct estimation rate for the smallest time dimension. As the time dimension increases at all levels of the break fraction factor, a decrease in 

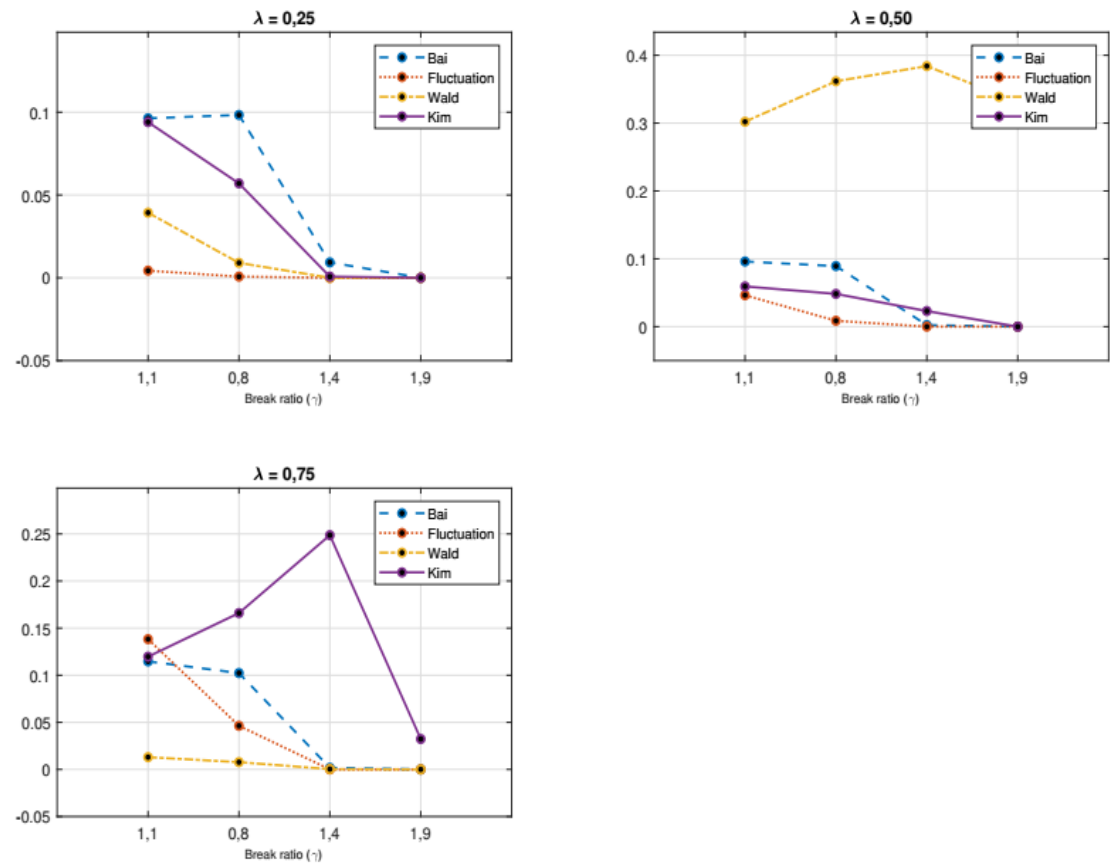

Figure 5. Simulation results for correct break point estimation rates $(N=32$ and $T=12)$

the correct estimation performance of the tests occurs and correct estimation rates approach to zero.

Nevertheless, the Bai test is the one affected least against time dimension. Thus, for medium and large time dimension, Bai Test has the highest correct break point estimation rate. Figure 7 shows the effects of the cross-section dimension on the correct break point estimation rates under the conditions that the break occurs in the middle of the series, and the mean of the series increases by $10 \%$ in postbreak. it is seen from Figure 7 that the increase in cross-section dimension have effect on the correct break point estimation rates of the methods on different ways. However, the length of the series forming the panel has a limited impact on the correct estimation rates for Bai Test, while other methods lead to a reduction in the correct estimates. Accordingly, the highest accurate estimation rates for the panels with short time series at these levels of the fraction section and fraction rate factors are reached with the Wald Test, while the highest rates of the other methods are reached with Bai Test as the time series length increases. Figure 8 shows the effects of the cross-section size under the conditions the break point in the third quarter of the series and the mean of the series increases by $10 \%$ after 

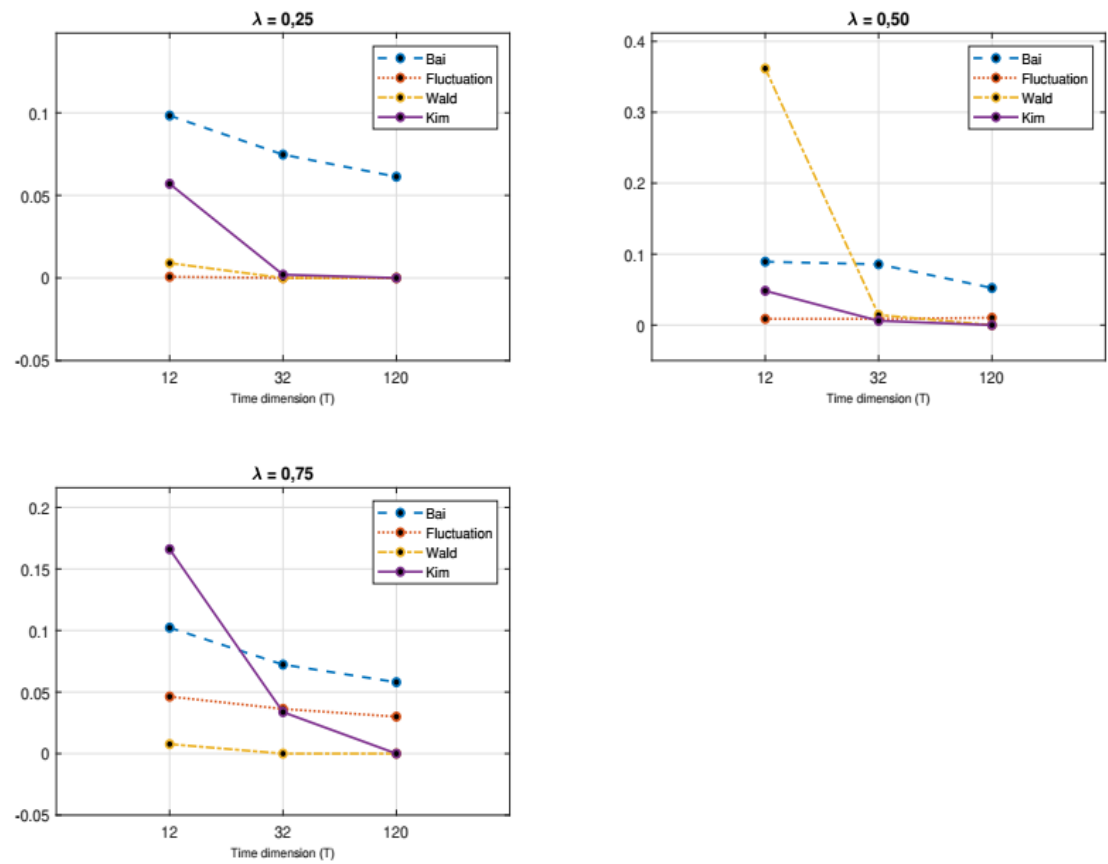

Figure 6. Simulation results for correct break point estimation rates $(\gamma=0,8$ and $N=32)$

break. It is seen in Figure 8 that increase in the cross section dimension have a different effects on the correct break point estimation rate of the methods. While the increase in cross-section size leads to a decrease in the correct break point estimation rate of the Fluctuation test, it leads to a slight increase in the correct estimation rate of Bai Test. However, the length of time has a different effect on the correct estimation rates of the methods and the correct estimation rates of the tests vary under different time dimension values. While changes in time dimension have a limited effect on the correct estimation rates of Bai Test, it leads to decrease in the correct estimation rates of the other methods. Accordingly, while the highest correct estimation rates in the panels with short time series at this level of the break fraction and the break ratio factors are reached with the Fluctuation Test, as the time lengths increase the highest correct estimation rates are reached with the Bai Test. Moreover, when Figures 3, 7 and 8 are evaluated altogether, it can be concluded that Bai Test has the highest correct estimation rates in the case where break occurs in the first quarter of the series, Wald Test has the highest correct estimation rates in the case where break occurs in the second quarter of the series 

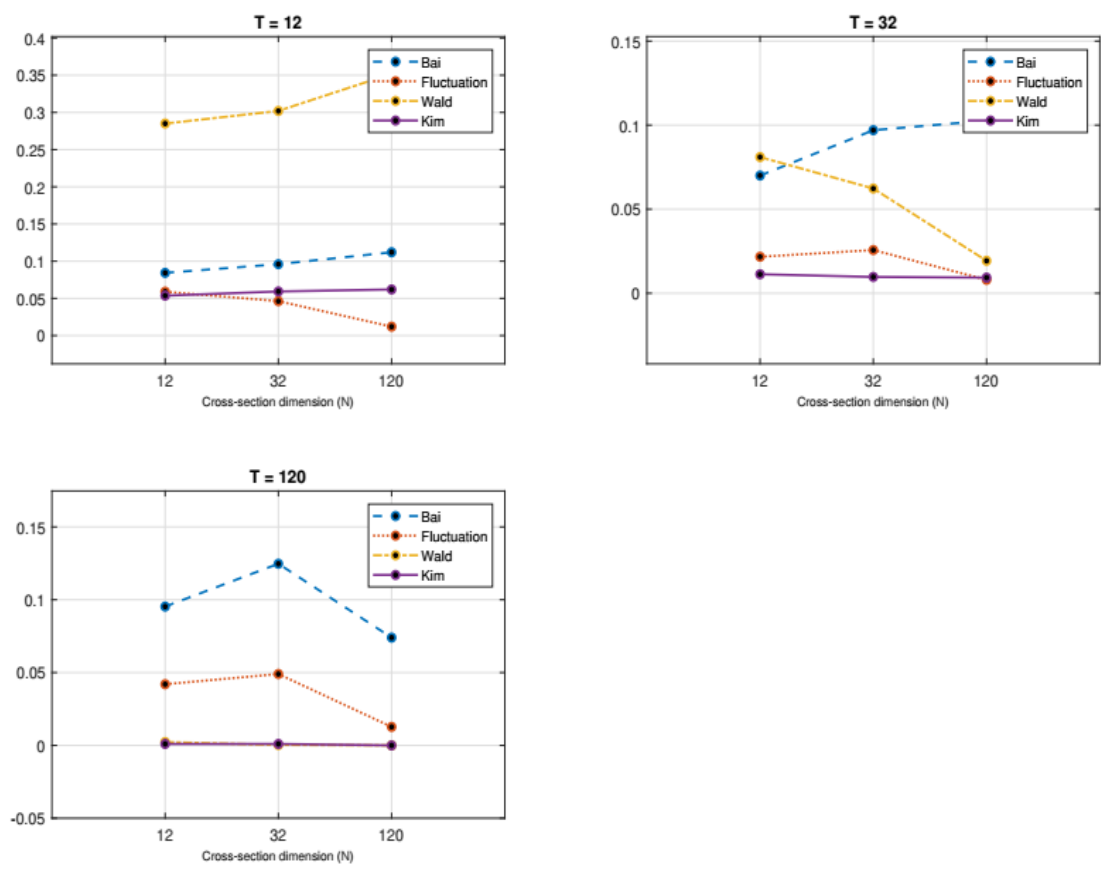

FigURE 7. Simulation results for correct break point estimation rates $(\gamma=1,1$ and $\lambda=0,5)$

and Fluctuation Test has the highest rate in the case where break occurs in the third quarter of the series.

However, while the level of the time dimension factor is 12, the Kim Test reveals the correct estimation rates similar to that of the Bai Test. Figure 9 concludes the effects of break ratio where a break occurs in the third quarter of the series by fixing time dimension at 32. From the Figure 9 it is seen that the correct estimation performance of the tests generally decreases as the break ratio increases. All methods can no longer accurately estimate the actual break point when postbreak means of the series are bigger at the rate of $\% 40$ or greater than pre-break means of the series. In addition, as the cross-section dimension increases, there is a limited decrease in the correct estimation performance of the tests. The Figure 10 shows that in the panel data with 32 section units and 12 time points, the ratio of the pre-break mean to the post-break mean and the part in which the break occurs are observed to have a significant effect on the correct estimation rates of the methods. While the highest correct break point estimation rates are reached with the Bai Test if the break occurs in the first quarter of the series, the Wald Test has the highest correct estimation rates in case the break occurs in the middle of 

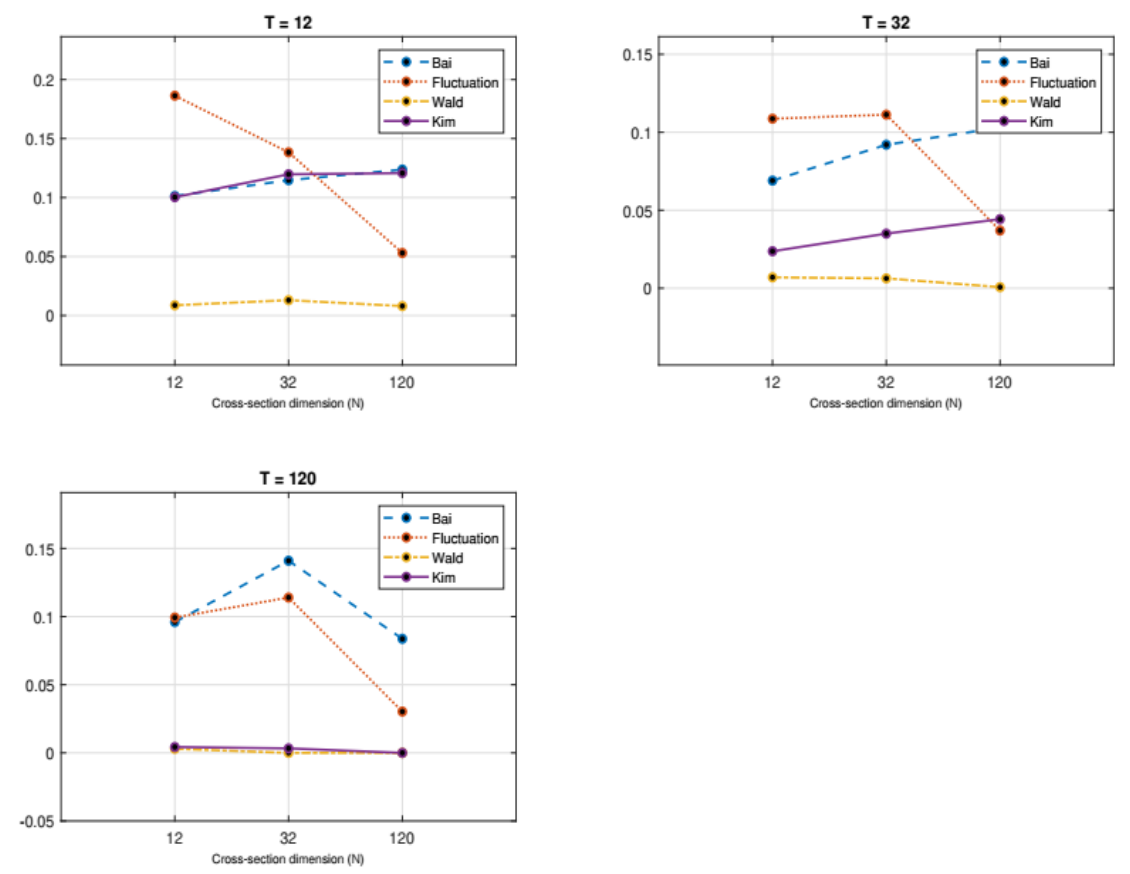

FigURE 8. Simulation results for correct break point estimation rates $(\gamma=1,1$ and $\lambda=0,75)$

the series. When the break occurs in the third quarter of the series, the Fluctuation Test has higher correct estimation rates if the change in the mean of the series is small. Nevertheless, the Kim Test reveals higher correct estimation rates if the change in the mean of the series is large. The Bai Test is the method of which the correct estimation performance is least affected by the changes of break fraction.

\section{Conclusion and Suggestions}

The correct estimation performance of the Bai, Fluctuation, Wald Statistics and Kim Tests, which are used to determine the structural break date in panel data, are examined via Monte Carlo simulations for the factors time dimension, cross-section dimension, break fraction and break ratio. The results can be concluded as follows:

- Bai Test has higher correct break point estimation rate than the other test methods except for some specific factor levels. If the break occurs in the first quarter of the series, the Bai Test shows a higher correct estimation performance. The Bai Test is the method that is least affected by the changes in the time dimension and the place on where the break point is. 

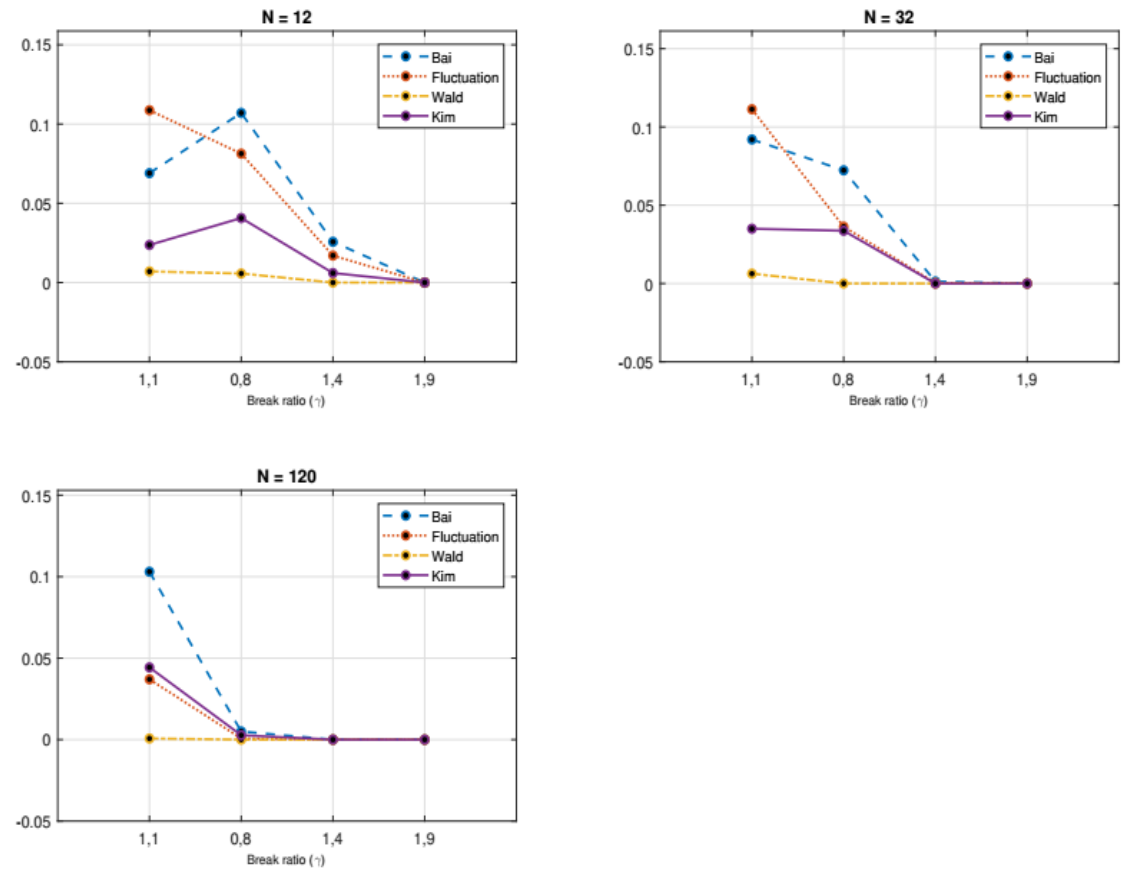

Figure 9. Simulation results for correct break point estimation rates $(T=32$ and $\lambda=0,75)$

- Wald Test has the highest correct estimation performance if the break occurs in the middle of the series. Nevertheless, the Bai Test shows a higher correct estimation performance if the break occurs in the middle of the series in panel data with large time dimensions since the changes in the time dimension have effects on the correct break point estimation rate of Bai and Wald Test on different ways.

- Correct estimation performance of the Fluctuation Test increases as the distance between the starting point of the series forming the panel and the break point increases. The Fluctuation Test shows a higher correct estimation performance when the break is in the third quarter of the series.

- The Kim Test shows a lower correct estimation performance than in other cases when the break is in the middle of the series. If the break occurs in the third quarter of the series, the Kim Test reveals a higher correct estimation performance. The Kim Test shows a higher correct estimation performance than the Fluctuation Test in the case when the break occurs in the third quarter of the series and where the time dimension of the panel is medium or large. 

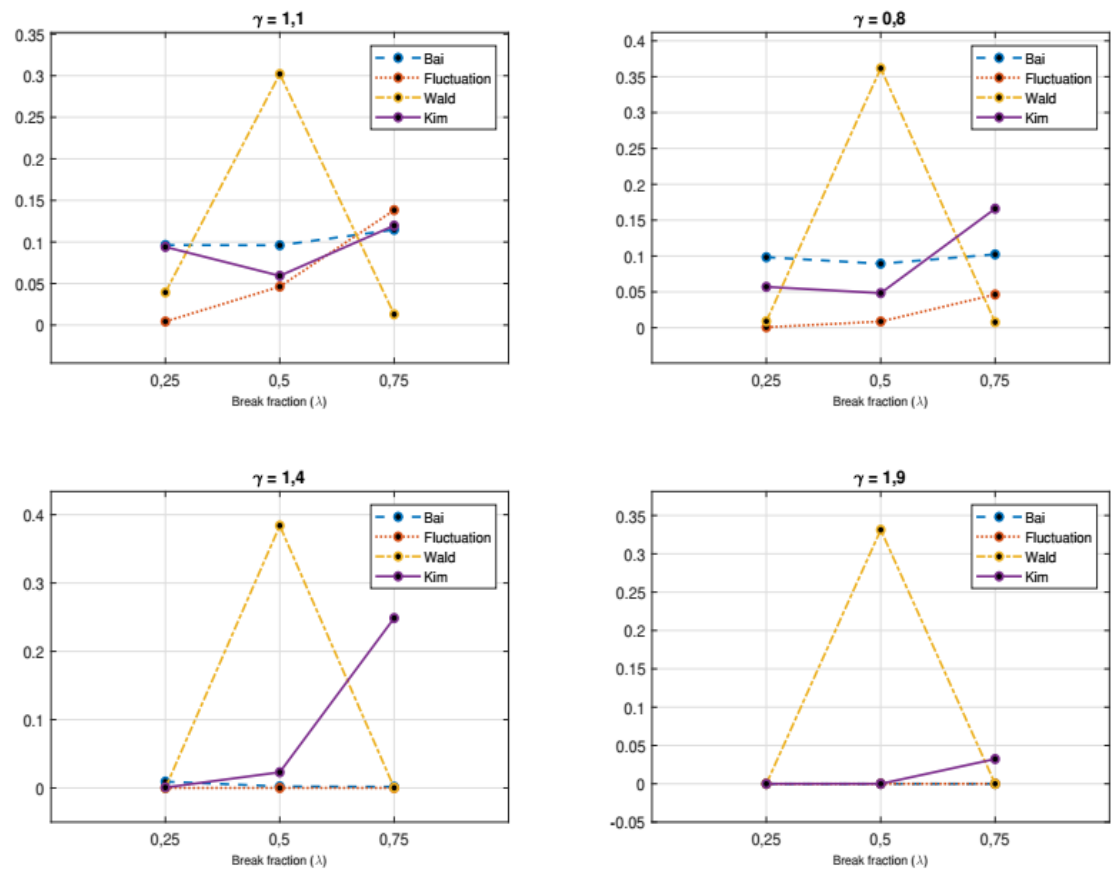

FigURE 10. Simulation results for correct break point estimation rates $(N=32$ and $T=12)$

- Mostly, increases in the cross-sectional dimension and/or time dimension have the effect on the correct estimation performance of the methods, which is slight but on decreasing direction. Nevertheless, the time dimension changes have a small effect on the Bai Test.

- Changes in the break ratio generally have a negative effect on the correct break point estimation performance of Bai, Fluctuation, Wald and Kim Tests. The methods can no longer correct estimate the actual break point when post-break mean is bigger $40 \%$ or more than the pre-break mean of the series.

- While the highest correct estimation rates are reached with the Bai Test in the case when the break occurs in the first quarter of the series, the highest correct estimation rates can be reached by the Wald Test if the break occurs in the middle of the series. In the case when the break occurs in the third quarter of the series, if the change in the mean of the series is small, the Fluctuation Test shows higher correct estimation rates, whereas in the case of a large change in the mean of the series, the Kim Test reveals 
higher correct estimation rates. The method of which correct estimation performance is less affected by the changes in the break fraction is Bai Test.

When evaluating the results of the tests concerning the correct break point estimation performance, it is seen that the correct estimation rates of the tests are usually adversely affected by the increase in the factor levels such as increase in the time or the cross-sectional dimensions. This may be due to a larger range of estimation values when the time dimension increases, or the fact that, when the cross-section dimension increases, the estimation of the break point estimates the same value as the standard error decreases. The results so far are the results obtained by evaluating test performances without considering the magnitude of the difference between the actual date of break and the break point estimate. However, it should be taken into consideration that the performance of the tests can be seen adverse since they do not estimate actual break date correctly at all, although they steadily lead very close estimation to the break point. On the other hand, we can only conclude that the tests have a good estimation performance since they have estimated the true break date only once although they generally produce very distant estimations to the actual break point.

\section{Concluding Remarks}

This paper investigates the performance of methods determining structural break point in a panel data with only one common break point at the time dimension. It is assumed that there is no serial correlation and/or cross section dependency. Also, the performance evaluation is performed with a consideration that there does not exist cross sectional heterogeneity. Although this study is limited for evaluation of performance of the methods assuming time and/or cross sectional dependency, it would be extended of the performance evaluation of these methods in terms of sensitivity or robustness for panel data set with time and/or cross sectional dependency.

The Monte Carlo simulations are based on the Equation 1 of the study which is the basic model of Bai (2010). Therefore, it could be expected that the Bai method or the methods which are compatible to this methodology such as Kim (2011) Model III has better performances more than others in this study. Since correct estimation performance of the methods have been investigated instead of comparing the performance of the considered tests in testing the null hypothesis, the data set is generated by considering only the model based on Equation 1 and the correct estimation rates of the methods were compared. Therefore, although data are produced according to the model proposed by Bai (2010), other methods have executed higher correct estimation performance than Bai (2010) and Kim (2011) at some factor levels. Thus, it has been possible to compare which test is more likely to correct estimate the break point according to the factor levels of the break point estimation methods considered in the study. Nevertheless, in the future studies, it 
may be useful to compare the correct estimation perspectives of the tests according to factor levels by considering the data generation processes based on other models.

\section{REFERENCES}

[1] Nelson, C. R. and Plosser, C. I., Trends and Random Walks in Macroeconomic Time Series: Some Evidence and Implications, Journal of Monetary Economics, 10 (1982), 139-162.

[2] Perron, P., The Great Crash, The Oil Price Shock and The Unit Root Hypothesis, Econometrica, 57 (6) (1989), 1361-1401.

[3] Zivot, E. and Andrews, D. W. K., Further Evidence on the Great Crash, the Oil-Price Shock, and the Unit-Root Hypothesis, Journal of Business 83 Economic Statistics, 10 (3) (1992).

[4] Lumsdaine, R. L. and Papell, D. H., Multiple Trend Breaks and the Unit-Root Hypothesis, The Review of Economics and Statistics, 79 (2) (1997), 212-218.

[5] Bai, J., Common Breaks in Means and Variances for Panel Data, Journal of Econometrics, 157 (2010), 78-92.

[6] Liao, W., Structural Breaks in Panel Data Models: A New Approach, Job Market Paper, 2008.

[7] Bai, J. and Perron, P., Estimating and Testing Linear Models with Multiple Structural Changes, Econometrica, 66, 1 (1998), 47-78.

[8] Carlion-i-Silvestre, J. L., Barrio-Castro, T. D. and Lopez-Bazo, E., Breaking the Panels: An application to the GDP per Capita, Econometrics Journal, 8 (2005), 159-175.

[9] Feng, Q., Kao, C. and Lazarova, S., Estimation and Identification of Change Points in Panel Models, Working Paper, Center for Policy Research, Syracuse University, Mimeo, (2008).

[10] Han, A. K. and Park, D., Testing for Structural Change in Panel Data: Application to a Study of U.S. Foreign Trade in Manufacturing Goods, The Review of Economics and Statistics, 71 (1) (1989), 135-142.

[11] Joseph, L. and Wolfson, D. B., Estimation in Multi-Path Change-Point Problems, Communications in Statistics-Theory and Methods, 21 (4) (1992), 897-913.

[12] Bai, J., Estimation of a Change Point in Multiple Regression Models, Review of Economics and Statistics, 79 (1997), 551-563.

[13] Bai, J., Lumsdaine, R. L. and Stock, J. H., Testing For and Dating Common Breaks in Multivariate Time Series, Review of Economic Studies Limited, 65 (1998), 395-432.

[14] Emerson, J. and Kao, C., Testing for Structural Change of a Time Trend Regression in Panel Data, Working Paper No. 15, Center for Policy Research, 137 (2000).

[15] Bai, J. and Perron, P., Computation and Analysis of Multiple Structural Change, Journal of Applied Econometrics, 18 (2003), 1-22.

[16] Kao, C., Trapani, L. and Urga, G., ,Modelling and Testing for Structural Changes in Panel Cointegration Models with Common and Idiosyncratic Stochastic Trend, Working Paper, Paper 73, Center for Policy Research, Surface, Syracuse University, 2007.

[17] Kim, D., Estimating a common deterministic time trend break in large panels with cross sectional dependence, Journal of Econometrics, 164 (2011), 310-330.

[18] Horváth, L. and Hušková, M. (2012). Change-Point Detection in Panel Data, Journal of Time Series Analysis, 33, 631-648.

[19] Chan, J., Horváth, L. and Hušková, M., Darling-Erdös Limit Results for Change-Point Detection in Panel Data, Journal of Statistical Planning and Inference, 143 (2013), 955-970.

[20] Li, F., Tian, Z., Xiao, Y. and Chen, Z., Variance Change-Point Detection in Panel Data Models, Economics Letters, 126 (2015), 140-143.

[21] Joseph, L. and Wolfson, D. B., Maximum Likelihood Estimation in the Multi-Path ChangePoint Problem, Annals of Institute of Statistical Mathematics, 45 (3) (1993), 511-530.

[22] Joseph, L., Vandal, A. C. and Wolfson, D. B., Estimation in the multipath change point problem for correlated data, The Canadian Journal of Statistics, 24 (1) (1996), 37-53. 
[23] Joseph, L., Wolfson, D. B., Berger, R. D. and Lyle, R. M., Change-Point Analysis of a Randomized Trial on the Effects of Calcium Supplementation on Blood Pressure, Bayesian Biostatistics, Berry, D. A. and Stangl, D. K. Eddition, Marcel Dekker Inc. 1996.

[24] Joseph, L., Wolfson, D. B., Berger, R. D. and Lyle, R. M. Analysis of Panel Data With Change-Points, Statistica Sinica, 7 (1997), 687-703.

[25] Dağlığlu, S, Bakır, M. A. Monte Carlo Evaluation of the Methods Estimating Structural Change Point in Panel Data. Sakarya University Journal of Science, 23 (3) (2019), 340-357. DOI: 10.16984 /saufenbilder.441235.

[26] Ploberger, W., Kramer, W. and Kontrus, K. A New Test for Structural Stability in the Linear Regression Model, Journal of Econometrics, 40 (1989), 307-318.

[27] Andrews, D. W. K., Tests for Parameter Instability and Structural Changes with Unknown Change Point, Econometrica, 61 (1993), 821-856.

[28] Andrews, D. W. K. and Ploberger, W., Optimal Tests When a Nuisance Parameter is Present Only under the Alternative, Econometrica, 62 (1994), 1383-1414.

[29] Perron, P., Zhu, X., Structural breaks with deterministic and stochastic trends, Journal of Econometrics, 129 (2005), 65-119.

Current address: Selim DAĞLIOĞLU (Corresponding author): Ministry of Family, Labour and Social Services, General Directorate of External Relations, Ankara Turkey

E-mail address: selim.daglioglu@gmail.com

ORCID Address: http://orcid.org/0000-0002-2006-8788

Current address: M. Akif BAKIR: Gazi University, Department of Statistics, Ankara Turkey

E-mail address: mabakir@gazi.edu.tr

ORCID Address: http://orcid.org/0000-0003-0774-0338 